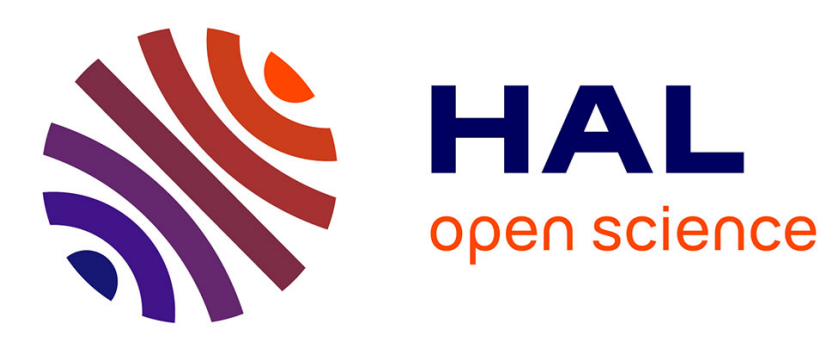

\title{
Continuous-wave diode-pumped solid-state laser with an intracavity fiber Bragg grating
}

Sylvie Yiou, Gaëlle Lucas-Leclin, François Balembois, Patrick Georges

\section{To cite this version:}

Sylvie Yiou, Gaëlle Lucas-Leclin, François Balembois, Patrick Georges. Continuous-wave diodepumped solid-state laser with an intracavity fiber Bragg grating. Applied optics, 2002, 41 (30), pp.6356-6359. hal-00700644

\section{HAL Id: hal-00700644 \\ https://hal-iogs.archives-ouvertes.fr/hal-00700644}

Submitted on 23 May 2012

HAL is a multi-disciplinary open access archive for the deposit and dissemination of scientific research documents, whether they are published or not. The documents may come from teaching and research institutions in France or abroad, or from public or private research centers.
L'archive ouverte pluridisciplinaire HAL, est destinée au dépôt et à la diffusion de documents scientifiques de niveau recherche, publiés ou non, émanant des établissements d'enseignement et de recherche français ou étrangers, des laboratoires publics ou privés. 


\title{
Continuous-wave diode-pumped solid-state laser with an intracavity fiber Bragg grating
}

\author{
Sylvie Yiou, Gaëlle Lucas-Leclin, Francois Balembois, and Patrick Georges
}

\begin{abstract}
We demonstrate the feasibility of a new concept of laser that benefits from both fiber and laser crystals: a cw diode-pumped solid-state laser with an intracavity fiber Bragg grating as the output coupler. The design of the laser cavity and the experimental demonstration are both described. Several applications are mentioned. (C) 2002 Optical Society of America

OCIS codes: $140.3480,140.3580,140.3530$.
\end{abstract}

\section{Introduction}

For many applications, there is a great interest toward the development of solid-state lasers. Indeed, bulk solid-state lasers (including bulk doped crystals or glasses) are attractive for several reasons. Many of these lasers can be pumped by laser diodes, allowing a compact assembly with a long lifetime. Moreover, they can deliver high-peak-power pulses, up to the gigawatt level. Emission wavelengths from bulk solid-state lasers associated with nonlinear crystals can cover a wide spectral region, from the ultraviolet to the mid-infrared range through the visible range. ${ }^{1}$

However, for many applications, such as telecommunications, spectroscopy, or medicine, it is also interesting to have a fibered output laser beam. Although fiber lasers make this property possible, they are still limited in their range of emission wavelengths, compared with bulk solid-state lasers. In particular, silica fibers suffer from inappropriate absorption for laser effect in the visible range. Laser effect has been observed from rare-earth ions in fluorozirconate fibers in this range, ${ }^{2}$ but such fibers unfortunately have many production and connecting problems and remain expensive.

A solution to benefit from both fiber and crystal lasers is to associate a crystal as the laser medium

The authors are with the Laboratoire Charles Fabry de L'Institute d'Optique, Unité Mixte de Recherche $8501 \mathrm{du}$ Centre National de la Recherche Scientifique, Centre Universitaire Paris XI, Bâtiment 503, 91403 Orsay, France. S. Yiou's email is sylvie.yiou@iota.u-psud.fr.

Received 30 April 2002; revised manuscript received 23 July 2002.

0003-6935/02/306356-04\$15.00/0

(C) 2002 Optical Society of America and the fiber as part of the laser cavity. Such a principle is commonly used with laser diodes. Fiber Bragg gratings enable the stabilization of the wavelength and the intensity of diode lasers for the pumping of erbium-doped fiber amplifiers. ${ }^{3,4}$ This principle also has been used to narrow the emission spectrum of a Cr:LiSAF laser in a plano-concave cavity. ${ }^{5}$ However, in both cases, the fiber Bragg grating does not act as the end mirror of the main laser cavity but is used as a coupled-cavity mirror in order to stabilize the laser emission by spectrally narrowed self-injection. Antireflection-coated diode lasers with a fiber Bragg grating as one end of the laser cavity have also been developed. ${ }^{6}$ Nevertheless, fiber Bragg gratings have never been used as conventional end mirrors with bulk solid-state lasers, to the best of our knowledge.

Our objective in this paper is to report the first demonstration of an intracavity fibered solid-state laser. The crystal that we used to demonstrate the feasibility of this laser is a well-known high-gain laser crystal, $\mathrm{Nd}: \mathrm{YVO}_{4}$. In this laser, the end mirror of the cavity is a fiber Bragg grating, and the output laser beam is then directly coupled in the optical fiber. The single-mode fiber realizes a spatial filter, and the laser beam is transversally single mode. (Note that in our application the fiber Bragg grating is not aimed toward the narrowing of the emission spectrum of the laser.)

\section{Definition of the Cavity}

The cavity design must take into account two constraints concerning the size of the laser beam radius. On the one hand, the size of the waist at the fiber input is imposed by the mode of the fiber, i.e., $3.1-\mu \mathrm{m}$ radius. On the other hand, as the laser rod is pumped by a laser diode, the radius of the laser mode 


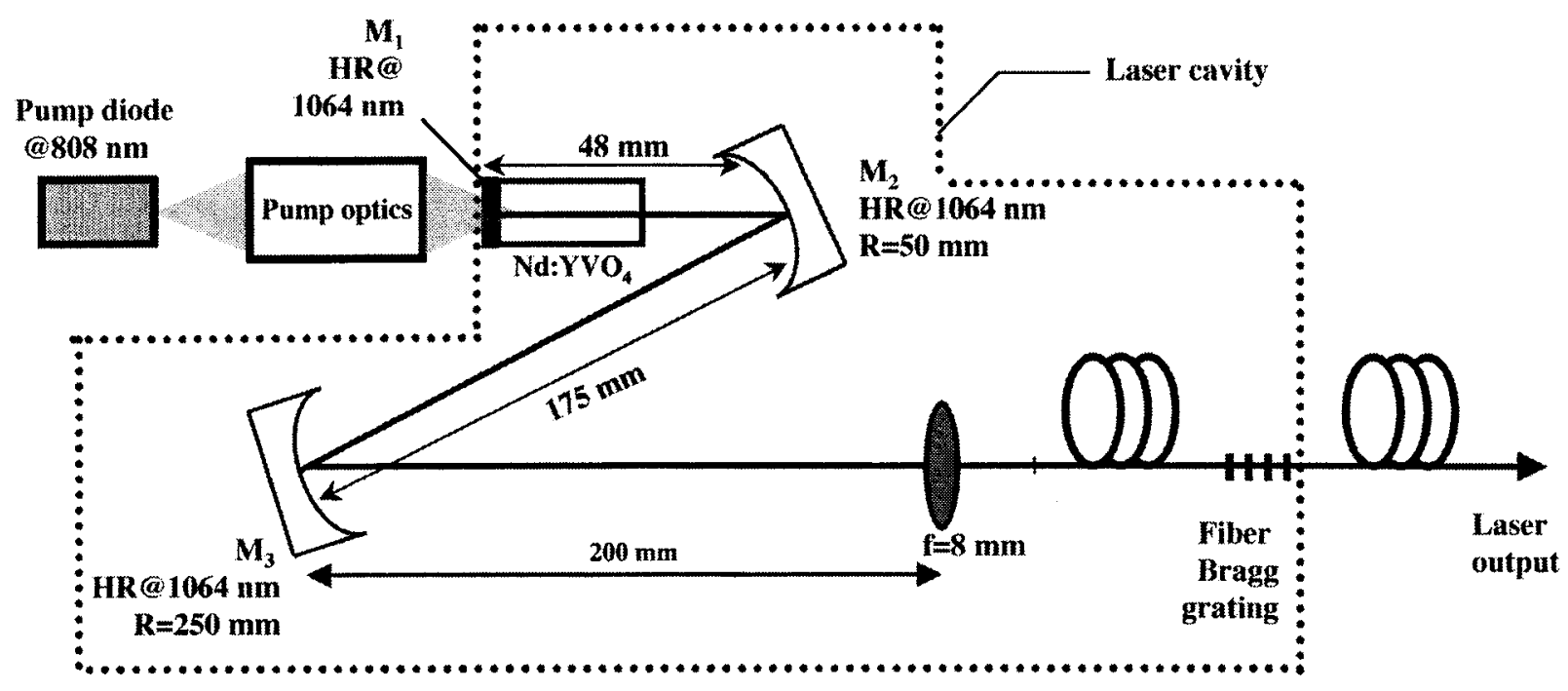

Fig. 1. Cavity design. HR, highly reflective.

in the crystal must be typically $\sim 100 \mu \mathrm{m}$ to optimize the overlapping between the pump and the laser beams in the crystal and to ensure an optimal gain. ${ }^{7}$

Therefore the cavity must have at least two waists; the size of the largest waist in the gain medium must be approximately 30 times the size of the smallest one at the end of the cavity. That is why a special cavity needs to be designed. Numerical simulations of cavities (using the ABCD matrix method for Gaussian beams) have led us to define the cavity described in Fig. 1 that satisfies both conditions. This is a fourmirror cavity with two folded concave mirrors $\mathrm{M}_{2}$ $\left(R_{c}=50 \mathrm{~mm}\right)$ and $\mathrm{M}_{3}\left(R_{c}=250 \mathrm{~mm}\right)$. An intracavity coupling objective with an 8-mm focal length focuses the light inside the optical fiber, and the fiber Bragg grating acts as the output coupler. The distances between the mirrors providing the appropriate beam sizes are reported in Fig. 1. In this configuration, the laser beam is slightly elliptical in the cavity owing to the tilt of the concave mirrors. The laser Gaussian mode radii inside the crystal were calculated to be 107 and $119 \mu \mathrm{m}$ in the tangential and sagittal directions, respectively. These values were obtained with an incidence angle of $10^{\circ}$ on mirror $\mathrm{M}_{2}$ and an incidence angle of $5^{\circ}$ on mirror $\mathrm{M}_{3}$, which correspond to our experimental conditions.

We have made preliminary experiments with an end mirror transmitting $10 \%$ of the light at $1064 \mathrm{~nm}$ instead of the fiber Bragg grating. On the one hand, our measurements have shown that the size of the beam waist at the end of the cavity was approximately $3 \mu \mathrm{m}$, corresponding to the mode-field radius of the fiber. On the other hand, the laser output beam was $\mathrm{TEM}_{00}$. At this point in our research we were led to think that the cavity design was suitable for our application.

\section{Experimental Setup}

We have chosen to demonstrate the principle of this intracavity fibered laser with a $\mathrm{Nd}: \mathrm{YVO}_{4}$ laser crystal because of its high gain, facilitating the introduction of intracavity elements. We used a $\mathrm{Nd}: \mathrm{YVO}_{4}$ laser rod with a $3-\mathrm{mm}$ diameter and a $3-\mathrm{mm}$ length. The $\mathrm{Nd}^{3+}$ concentration of the laser crystal was 1 at. $\%$. The crystal was oriented with the $a$ axis along the rod axis. One face was highly reflective at $1064 \mathrm{~nm}$ and highly transmittive at $808 \mathrm{~nm}$, and the other face had an antireflection coating at $1064 \mathrm{~nm}$. The crystal was placed on a copper mounting with no special cooling. The pump source was a laser diode emitting a maximal output power of $1.4 \mathrm{~W}$ at $808 \mathrm{~nm}$ with a $1 \mu \mathrm{m} \times 100 \mu \mathrm{m}$ emitting area. The pump beam was collimated with an aspherical lens of $4.5-\mathrm{mm}$ focal length, then reshaped in the fast axis through an afocal system consisting of a cylindrical positive lens $(f=150 \mathrm{~mm})$ and a cylindrical negative lens $(f=-25.4 \mathrm{~mm})$. The pump beam was then focused inside the laser rod by means of an 8-mm-focal-length objective. These pump optics provided a magnification of 10.5 in the direction perpendicular to the diode junction and a magnification of 1.77 in the direction parallel to the diode junction. So the pump beam size at the focal point in the crystal was approximately 10 $\mu \mathrm{m} \times 200 \mu \mathrm{m}$. The transmission of this system was approximately $80 \%$. The temperature of the diode was stabilized at $20^{\circ} \mathrm{C}$, corresponding to the maximum absorption of the crystal (almost 98\%) and held constant during all of the following experiments.

The mirrors $\mathrm{M}_{2}$ and $\mathrm{M}_{3}$ were both highly reflective at $1064 \mathrm{~nm}$. The coupling objective of $8-\mathrm{mm}$ focal length (Melles-Griot, 06 GLC 002) had a transmission of $95 \%$ at $1064 \mathrm{~nm}$; it was aberration free for the infinite/focus conjugation.

The optical fiber (Corning SMF 1060) was single mode at $1064 \mathrm{~nm}$, with a 920-nm cut-off wavelength. Its mode field radius was $3.1 \mu \mathrm{m}$ at $1060 \mathrm{~nm}$, and its numerical aperture was 0.14. Both fiber ends were cleaved at an $8^{\circ}$ angle (Fiber Connector-Angle Polished Cleaved connectors) to avoid any parasitic laser oscillation, but they were not antireflection coated. The 


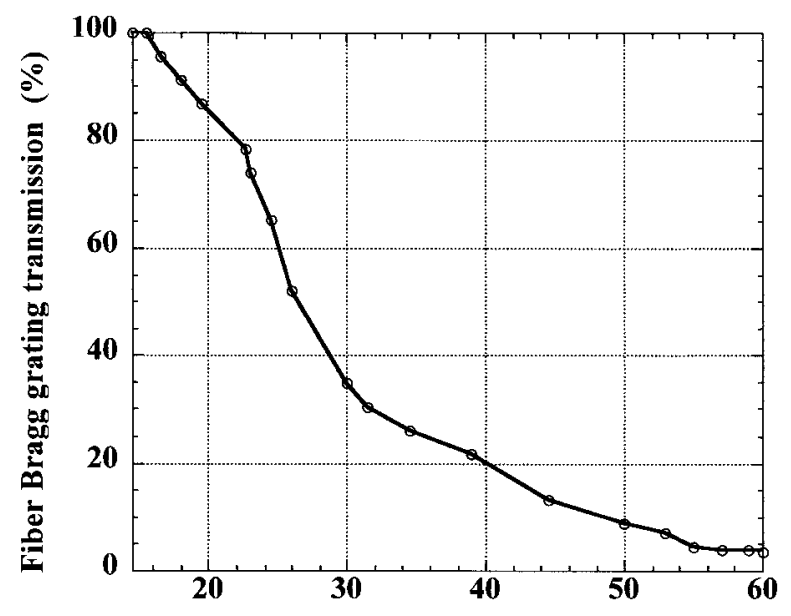

Fiber Bragg grating temperature $\left({ }^{\circ} \mathrm{C}\right)$

Fig. 2. Transmission of the fiber Bragg grating versus its temperature $\left({ }^{\circ} \mathrm{C}\right)$ at $1064.01 \mathrm{~nm}$.

length of the fiber was about $2 \mathrm{~m}$, and the fiber Bragg grating was written in its center. The length of the grating was approximately $1 \mathrm{~cm}$. The central wavelength of the grating was $1063.82 \mathrm{~nm}$; at this wavelength, the reflectivity was $98.1 \%$, and the FWHM was $0.72 \mathrm{~nm}$. The fiber grating was placed on a copper mounting with a temperature stabilization.

The center wavelength of the Bragg grating $(1063.82 \mathrm{~nm})$ could be temperature tuned at the rate of approximately $7 \mathrm{pm} /{ }^{\circ} \mathrm{C} .8$ This enabled us to continuously vary the transmission of our grating at $1064.01 \mathrm{~nm}$ (corresponding to the $\mathrm{Nd}: \mathrm{YVO}_{4}$ emission wavelength) from $4 \%$ to $100 \%$ by modifying the grating temperature from $60{ }^{\circ} \mathrm{C}$ to $10{ }^{\circ} \mathrm{C}$, respectively. Figure 2 shows the change in transmission with respect to the temperature of the grating at 1064.01 $\mathrm{nm}$. The stability of the temperature regulation was approximately $\pm 1{ }^{\circ} \mathrm{C}$ during these measurements. The curve in the figure shows that the fiber Bragg grating could play the role of an output coupler whose transmission could be varied continuously with its temperature. This property will enable us to experimentally find the optimal output coupling.

\section{Laser Operation}

Figure 3 illustrates the search for the optimal output coupler through a tuning of the grating temperature. The laser output power was measured with a silicium photodetector placed at the output end of the fiber. The optimal output coupler corresponded to the maximal output power at 1.1-W absorbed pump power. This maximal output power was found for a grating temperature of $41^{\circ} \mathrm{C}$, i.e. a grating transmission of 18\%. Figure 4 shows the evolution of the laser output power with respect to the absorbed pump power at this grating temperature. We have optimized laser operation at the maximal pump power (i.e., $1.4 \mathrm{~W}$ of incident power) and then decreased the pump power. The laser reached threshold at an absorbed pump power of $150 \mathrm{~mW}$. We obtained an output

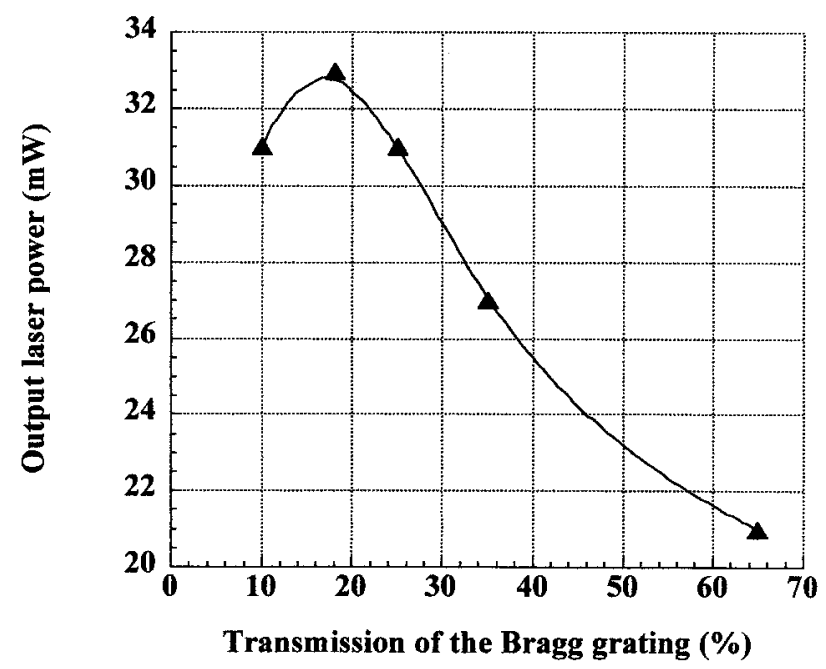

Fig. 3. Output power versus the transmission of the Bragg grating. The absorbed pump power is $1.1 \mathrm{~W}$; the temperature of the grating is varied from $50^{\circ} \mathrm{C}$ to $24^{\circ} \mathrm{C}$.

power of $39 \mathrm{~mW}$ for an absorbed pump power of 1.1 $\mathrm{W}$, and the laser output beam was TEM $\mathrm{TO}_{00}$. The dip in laser output power centered around $650 \mathrm{~mW}$ of absorbed pump power is certainly due to thermal effects. Through simulations we estimated that the thermal lens varied from approximately $48 \mathrm{~cm}$ at 150 $\mathrm{mW}$ of absorbed pump power to $6 \mathrm{~cm}$ at $1.1 \mathrm{~W}$ of absorbed pump power. ${ }^{9}$ This variation slightly modifies the spot size at the entrance of the fiber from 2.8 to $3.1 \mu \mathrm{m}$, respectively. Laser operation is then affected, since there was no change in the adjustments after the optimization of the output power at the maximal pump power. The relatively low (42\%) slope efficiency obtained is due both to the astigmatism introduced by concave mirrors $\mathrm{M}_{2}$ and $\mathrm{M}_{3}$ reducing the coupling into the fiber and to the

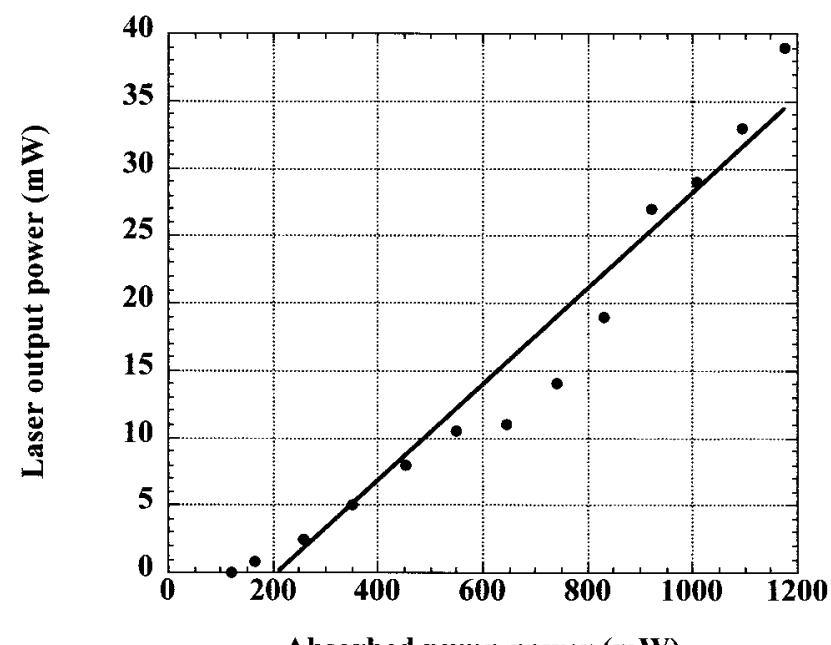

Absorbed pump power $(\mathrm{mW})$

Fig. 4. Laser output power at $1064.01 \mathrm{~nm}$ versus absorbed pump power. The transmission of the output coupler is $18 \%$, corresponding to a temperature of the grating of $41^{\circ} \mathrm{C}$. The slope efficiency is $4.2 \%$. 
intracavity losses (see next paragraph). Moreover, the beam radius in the crystal is very sensitive to the distance between the coupling objective and the input end of the fiber. Consequently, we assume that the effective size of the laser mode in the crystal is not exactly appropriate to the pump mode. However we recall that the purpose of the present article was to report the feasibility of this laser. That is why the laser output characteristics have not been optimized at present. Nevertheless, it is interesting to identify the passive losses to improve the performances of the laser in the future.

First we estimated the passive losses in the cavity that were not due to the fiber with the Findlay-Clay analysis. ${ }^{10}$ We successively replaced the fiber by two different plane mirrors placed at the focus point of the coupling objective. In the two cases we measured the absorbed pump power at threshold $P_{\text {th }}$. Indeed, $P_{\text {th }}$ is proportional to $L+T$, where $L$ represents the passive double-pass losses and $T$ is the transmission of the output coupler. Whereas $P_{\text {th }}$ was $300 \mathrm{~mW}$ for $T=8 \%$ and $330 \mathrm{~mW}$ for $T=10 \%$, we deduced that the passive losses $L$ were approximately $12 \%$ (including the 10\% double-pass losses for the 8-mm coupling objective).

Then we estimated the double-pass losses $L_{f}$ in the fibered cavity by measuring the absorbed pump power at threshold for two different temperatures of the grating $T_{B}$, i.e. for two different transmissions $T^{\prime}$ of the output coupler. We measured $P_{\text {th }}$ for $T_{B}=$ $25^{\circ} \mathrm{C}$ and at $T_{B}=42{ }^{\circ} \mathrm{C}$, i.e. for $T^{\prime}=61 \%$ and $T^{\prime}=$ $17 \%$ (200 $\mathrm{mW}$ and $100 \mathrm{~mW}$, respectively). The passive losses $L_{f}$ were therefore approximately $28 \%$. This value takes into account the $12 \%$ double-pass losses of the laser cavity without the fiber and the $8 \%$ double-pass losses due to the uncoated fiber. Therefore the other losses due to the fiber are approximately $8 \%$ and are supposed to be leaked in the cladding (the fiber was slightly damaged at one point) or induced by the slightly elliptical beam propagating in the cavity.

\section{Conclusion and Applications}

We have demonstrated the feasibility of a diodepumped solid-state laser with a fiber Bragg grating as the output coupler, for the first time, to our knowledge. The output beam of this laser is coupled directly in the optical fiber, similarly to a fiber laser. This laser requires a cavity with two waists of very different sizes, one in the gain medium and the other at the input end of the fiber. With a high-gain $\mathrm{Nd}$ : $\mathrm{YVO}_{4}$ crystal as the amplifying medium and a fourmirror cavity with an intracavity objective (the fourth mirror being the Bragg grating), laser operation was obtained. The output power was $39 \mathrm{~mW}$ at $1064 \mathrm{~nm}$ for an absorbed pump power of $1.1 \mathrm{~W}$, and the output beam was $\mathrm{TEM}_{00}$. Although the laser efficiency was not optimized, several guidelines will enable us to enhance the performances of this laser. First, antireflection coatings of the ends of the fiber could reduce the losses due to the fiber. Moreover, other types of cavities should be studied, leading to less astigmatism in the cavity and providing a better slope efficiency. For example, cavities with only one concave mirror and an objective with a smaller focal length might be easier to align.

The tunability of the Bragg grating wavelength is advantageous for laser sources, as it is quite easy to control the temperature of the grating. An everwider range of central wavelength can be reached through application of a mechanical tension to the grating. In our experiment, in which the emission wavelength is imposed by the laser crystal, the fiber Bragg grating plays the role of an output coupler with a transmission that varies continuously with its temperature. Since fiber Bragg gratings could be manufactured at any wavelength, similar lasers could be realized with other crystals. In addition, if one uses a crystal with a broad emission spectrum, the fiber Bragg grating can easily control the emission wavelength to manage a widely tunable laser source with a fibered output.

Moreover, an intracavity fiber could enable one to make very long cavities, which are difficult to achieve in free space and require, for example, a multiplepass cavity. ${ }^{11}$

We acknowledge the NetTest company and the Centre National de la Recherche Scientifique for the financial support of S. Yiou's Ph.D.

\section{References}

1. A. A. Kaminskii, Laser Crystals (Springler-Verlag, New York, 1981).

2. J. Y. Allain, M. Monerie, and H. Poignant, "Blue upconversion fluorozirconate fibre laser," Electron. Lett. 26, 166-168 (1990).

3. R. Kashyap, "Fiber grating lasers and amplifiers," in Fiber Bragg Gratings, P. L. Kelly, I. Kaminov, and G. Agrawal, eds., Optics and Photonics Series (Academic, San Diego, Calif., 1999).

4. B. F. Ventrudo, G. A. Rogers, G. S. Lick, D. Hargreaves, and T. N. Demayo, "Wavelength and intensity stabilisation of 980-nm diode lasers coupled to fibre Bragg gratings," Electron. Lett. 30, 2147-2148 (1994).

5. N. J. Vasa, T. Okada, M. Maeda, T. Mizunami, and O. Uchino, "Single-mode cw $\mathrm{Cr}^{3+}: \mathrm{LiSrAlF}_{6}$ laser using an external fiber grating," Opt. Lett. 21, 1472-1473 (1996).

6. P. A. Morton, V. Mizrahi, T. Tanbun-Ek, R. A. Logan, P. J. Lemaire, H. M. Presby, T. Erdogan, S. L. Woodward, J. E. Sipe, M. R. Phillips, A. M. Sergent, and K. W. Wecht, "Stable single mode hybrid laser with high power and narrow linewidth," App. Phys. Lett. 64, 2634-2636 (1994).

7. P. Laporta and M. Brussard, "Design criteria for mode size optimization in diode-pumped solid-state lasers," IEEE J. Quantum Electron. 27, 2319-2326 (1991).

8. K. O. Hill, Y. Fujii, D. C. Johnson, and B. S. Kawasaki, "Photosensitivity in optical fiber waveguides: application to reflection filter fabrication,” App. Phys. Lett. 32, 647-649 (1978).

9. Y. F. Chen and S. W. Tsai, "Diode-pumped $Q$-switched Nd: $\mathrm{YVO}_{4}$ yellow laser with intracavity sum-frequency mixing," Opt. Lett. 27, 397-399 (2002).

10. D. Findlay and R. A. Clay, "The measurement of internal losses in 4-level lasers," Phys. Lett. 20, 277-278 (1966).

11. S. H. Cho, X. Kärtner, U. Morgner, E. P. Ippen, J. G. Fujimoto, J. E. Cunningham, and W. H. Knox, "Generation of 90-nJ pulses with a $4-\mathrm{MHz}$ repetition-rate Kerr-lens mode-locked Ti: $\mathrm{Al}_{2} \mathrm{O}_{3}$ laser operating with net positive and negative intracavity dispersion," Opt. Lett. 26, 560-562 (2001). 